(C) 2000 International Press

Adv. Theor. Math. Phys. 6 (2000) 1893-1908

\title{
On Open/Closed String
}

\section{Duality}

\author{
Justin Khoury and Herman Verlinde
}

Joseph Henry Laboratories, Princeton University

Princeton, NJ 08544, USA

jkhoury@princeton.edu

verlinde@feynman.princeton.edu

\begin{abstract}
It was recently shown, using the AdS/CFT correspondence, that the low energy effective action of a large $N$ open string theory satisfies a holographic RG flow equation closely related to the Hamilton-Jacobi equation of 5-d supergravity. In this paper we re-obtain the same flow equation in the dual regime of small 't Hooft coupling $\lambda \ll 1$. Our derivation makes use of the conformal equivalence between planar open string diagrams and closed string tree diagrams. This equivalence can be viewed as a microscopic explanation of the open/closed string duality that underlies the AdS/CFT correspondence.
\end{abstract}

e-print archive: http://xxx.lanl.gov/hep-th/0001056 


\section{Introduction}

In this paper we will consider the world volume theory of a set of $N$ D3 branes, in the limit of large $N$, at finite $\alpha^{\prime}$. When embedded in flat 10-d space-time, the theory reduces at low energies to $\mathcal{N}=4$ supersymmetric Yang-Mills theory. More generally, we can deform the model by turning on gauge invariant couplings $\phi^{i}$ and consider the quantum partition function as a function of the $\phi^{i}$

$$
\exp (i \Gamma(\phi))=\left\langle\exp \left(i \int \phi^{i} O_{i}\right)\right\rangle
$$

Via the famous AdS/CFT correspondence [1], this partition function has, for $\alpha^{\prime} \rightarrow 0$ and for large 't Hooft coupling $\lambda=N g_{y m}^{2}$, a dual represention as that of IIB string theory on (a deformation of) $A d S_{5} \times$ $S^{5}$. In this correspondence, the couplings $\phi^{i}$ - which among others include the dilaton, 4-d space-time metric, as well as RR fields - specify the non-dynamical asymptotic values of the corresponding set of closed string fields [2] [3]. When the $\phi^{i}$ represent finite expectation values, they generally break the conformal invariance and part or all of the supersymmetry of the low energy theory.

An interesting generalization of this duality arises when all directions perpendicular to the D3-branes are taken to be compact [4]. In this case one can not take the decoupling limit and interactions between open and closed strings will remain relevant. Instead, the target space of the IIB string theory is described by a warped compactification similar to the Randall-Sundrum geometry [5], and variations of the couplings $\phi^{i}$ correspond to normalizable, and thus dynamical, fluctuations of closed string modes. The low energy dynamics of these modes is described by an effective action $S(\phi)$, that includes besides the nonlocal induced action $\Gamma(\phi)$ also a local contribution $S_{E}(\phi)$ arising from the $\mathrm{KK}$ reduction of the 10-d effective action of the IIB string theory

$$
S(\phi)=S_{E}(\phi)+\Gamma(\phi) .
$$

In [6] some properties of this effective action where studied for large $\lambda$ with the help of the AdS/CFT correspondence. Using the dual supergravity approximation and elementary results of Hamilton-Jacobi theory, it was found that in this regime $S(\phi)$ satisfies an RG flow equa- 
tion of the following schematic form

$$
\beta^{i}(\phi) \frac{\partial S}{\partial \phi^{i}}-\frac{1}{2} G^{i j} \frac{\partial S}{\partial \phi^{i}} \frac{\partial S}{\partial \phi^{j}}=0
$$

Here $G^{i j}$ denotes some apropriate metric on the space of couplings, and $\beta^{i}(\phi)$ are 'beta-functions' that describe the classical flow velocities of the $\phi$-fields as a function of the holographic extra dimension. They are expressed in terms of the local action $S_{E}(\phi)$ as

$$
\beta^{i}(\phi)=G^{i j} \frac{\partial S_{E}}{\partial \phi^{i}}
$$

For a more detailed explanation of these relations we refer to [6].

In [6] eqn (3) was interpreted as an RG invariance of the total gravitational effective action $S$, in the sense that any classical extremum of $S$ automatically lies on a complete RG trajectory of classical solutions connected by the flow relation

$$
\dot{\phi}^{i}=\beta^{i}(\phi) .
$$

This property of $S$, if true in general, could be of particular importance for the cosmological constant problem. The derivation of (3) as given in [6], however, is valid only in the limit of large $N$ and large 't Hooft coupling $\lambda \gg 1$. It is important therefore to investigate whether these relations can be generalized and extended to other regimes as well. In the following we will concentrate on the dual regime of small $\lambda$, but still infinite $N$. We will find the positive result that the exact same equations (2), (3) and (4) remain valid also in this regime, except that the quantities $\Gamma, S_{E}, G^{i j}$ and $\beta^{i}$ are now all defined via their weak coupling descriptions.

The main idea behind our derivation is that the world sheet of a planar multi-loop diagram in open string theory is conformally equivalent to a closed string tree diagram. Indeed, all holes in the open string diagram can be represented in the dual channel by means of external closed string states, equal to the appropriate D-brane boundary state $|B\rangle$. Via this dual representation all potential UV divergences of the open string diagram become equivalent to potential IR divergences due to on-shell closed string states in the dual channel. It is not too surprising therefore that the RG structure of the large $N$ open string theory 
can be made to look identical to a classical evolution equation of closed string theory.

Several elements in our reasoning have appeared in earlier works. We mention here:

- The Fischler-Susskind mechanism for cancelling string loop divergences [7]; for a recent discussion of the FS-mechanism in relation with D3-brane physics, see [8].

- The interpretation given in [9] of the BV symmetry of closed string field theory [10] as an 'exact' RG invariance à la Polchinski [11].

- The non-linear flow relation, proposed by Polyakov in [12], satisfied by the tree level partition function in a general non-critical string theory.

In a pure field theory context, the structure described below also seems closely related to the old recursive formula for QFT counterterms due to Bogolyubov [13], that forms the basis for the classic BPHZ renormalization method. We will comment on this correspondence (which may prove useful for taking the $\alpha^{\prime} \rightarrow 0$ limit of our result) in section 3 . In section 4 we summarize our conclusions.

\section{Derivation of the flow equation}

For small 't Hooft coupling $\lambda$, the total low energy effective action $S(\phi)$ is obtained by summing over all n-loop planar open string diagrams in the closed string background specified by $\phi$. Schematically ${ }^{1}$

$$
S(\phi)=\Gamma_{0}(\phi)+\sum_{\mathrm{n} \geq 1} \lambda^{n} \Gamma_{\mathrm{n}}(\phi)
$$

\footnotetext{
${ }^{1}$ Here we are using the same notation $O_{i}$ for the closed string vertex operators dual to the fields $\phi^{i}$, as used in eqn (1) for the gauge theory operators dual to $\phi^{i}$. In principle, one can make a one-to-one correspondence between the two, by comparing the 2-d world-sheet action with the D3-brane world volume action in a given closed string background.
} 


$$
\Gamma_{\mathrm{n}}(\phi)=\left\langle\exp \left(i \int \phi^{i} O_{i}\right)\right\rangle_{\mathrm{n}}
$$

This term $\Gamma_{n}(\phi)$ is the $\mathrm{n}$-1-loop open string contribution, given by the partition function of the world-sheet sigma-model (parametrized by $\phi$ ) on a sphere with $\mathrm{n}$ holes, integrated over all moduli parameterizing the relative sizes and locations of these holes. Both the sigma-model expectation value and the integral over these moduli may produce potentially infinite answers, which both can be regularized by introducing an explicit cut-off scale $\epsilon$. We will give a concrete prescription for this cut-off momentarily. In any case, in the end all physical answers should, when expressed in terms of renormalized couplings, be independent of this cut-off. In particular, writing

$$
S(\phi(\epsilon) ; \epsilon),
$$

where $\phi(\epsilon)$ is the renormalized sigma-model background satisfying the $\mathrm{RG}$ equation

$$
\epsilon \frac{\partial \phi^{i}}{\partial \epsilon}=\beta^{i}(\phi)
$$

with $\beta^{i}(\phi)$ the sigma model beta-functions, we must require that the total $\epsilon$ dependence cancels

$$
\epsilon \frac{d S}{d \epsilon}=\beta^{i}(\phi) \frac{\partial S}{\partial \phi^{i}}+\epsilon \frac{\partial S}{\partial \epsilon}=0
$$

This requirement reduces to the usual condition of conformal invariance in the limit $\lambda \rightarrow 0$, when the holes of the open string loops are absent. The condition (10) with $\lambda>0$ relates divergences arising from deviation from world-sheet conformal invariance to divergences coming from the shrinking of open string loops; the cancellation of these two different types of divergences is known as the Fischler-Susskind mechanism [7].

To implement this cancellation mechanism, we need a sufficiently precise definition of the cut-off $\epsilon$, both for the divergences of the sigmamodel as well as for regulating the moduli integral. For this we will make use of some technology from closed string field theory [10]. To any Riemann surface (with holes) with given conformal structure, we can assign a unique minimal area metric $g_{\alpha \beta}$. For a given point on the moduli space of the open string loop diagram, we can thus measure the minimal geodesic length $\ell(C)$, as defined using this minimal area metric, of all non-contractible contours $C$ surrounding a non-zero number of 
holes. (See fig 1a). The UV divergences of the loop diagram arise when one or more of these geodesic lengths $\ell(C)$ tends to zero. We will therefore introduce a UV regulator $\epsilon$ by requiring that the moduli integral is restricted to those conformal structures for which

$$
\ell(C) \geq \epsilon
$$

for all non-contractible contours $C$. Hence the boundary of the regulated moduli space are degenerate surfaces for which the above bound (11) is saturated for one or more contours $C$.
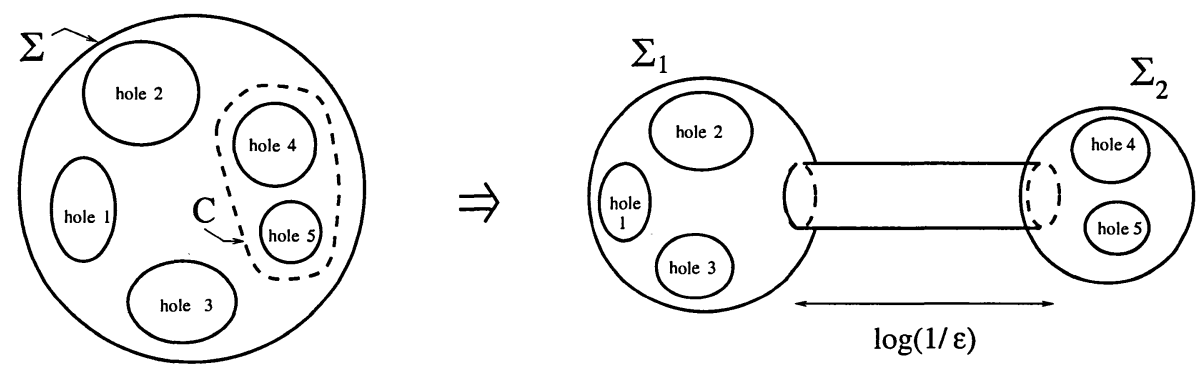

Fig. 1a and 1b. A planar n-loop open string diagram is given by the integral over all shapes of a spherical Riemann surface $\Sigma$ with $n+1$ holes. When a non-contractible contour $C$ surrounding some of the holes acquires a very small length $\ell(C)=\epsilon$, the surface degenerates into two separate spherical surfaces $\Sigma_{1}$ and $\Sigma_{2}$ connected by a long tube of length $\log (1 / \epsilon)$.

Since in the end we need to compare this type of degeneration of the open string loop diagram with the sigma-model divergences, it seems most practical to regulate the sigma-model expectation values in an analogous fashion. To this end, we explicitly expand the exponential in eqn (7)

$$
\Gamma_{\mathrm{n}}(\phi ; \epsilon)=\sum_{k \geq 0} \frac{1}{k !}\langle\underbrace{\Phi \cdots \Phi}_{k \times}\rangle_{\mathrm{n}}=\sum_{k \geq 0} \frac{1}{k !}\left\langle(\Phi)^{k}\right\rangle_{\mathrm{n}}
$$

with

$$
\Phi=\sum_{i} \int \phi^{i} O_{i}
$$

The $k$-th order term on the right-hand side is a correlator, defined in the $\phi=0$ sigma-model, of $k$ operators $\Phi$ on an $(n-1)$ loop open 
string diagram. The resulting amplitude is therefore an integral over the moduli space of a sphere with $n$ holes and $k$ punctures. (See fig 2a.) We can now apply the same construction as above, and use the unique minimal area metric on this punctured surface to assign a given minimal geodesic length to all closed contours surrounding a non-zero number of holes and/or punctures, and require that all such lengths must be larger than the cut-off $\epsilon$. In this way we have indeed introduced one uniform cut-off procedure for both types of divergences. ${ }^{2}$

The above equation (12) in fact needs some extra specification for the case $n=0$. On the sphere without holes one needs, due to the invariance under the global conformal group $S L(2, C)$, at least three operator insertions $\Phi$ to obtain a well-defined expectation value. To write the $n=0$ contribution to the effective action, we must thus include a separate kinetic term via

$$
\Gamma_{0}(\phi ; \epsilon)=\frac{1}{2}\langle\Phi|Q| \Phi\rangle+\sum_{k \geq 3} \frac{1}{k !}\left\langle(\Phi)^{k}\right\rangle
$$

Here $Q$ denotes the nilpotent world-sheet BRST charge of the $\phi=0$ sigma-model and $|\Phi\rangle=\sum_{i} \phi^{i}\left|O_{i}\right\rangle$ is the state corresponding to the sigma-model background $\Phi$. The above expression for $\Gamma_{0}(\phi)$ is of the same form as the standard classical action of closed string field theory (for a detailed discussion of its definition and properties, see [10]). Note, however, that in the present context the classical equations of motion of $\phi$ must be derived from the total action $S(\phi)$ given in (6), and not from just $\Gamma_{0}(\phi)$. Indeed, since $\Gamma_{0}(\phi)$ has no contribution from surfaces with holes, it does not have any direct knowledge of the presence of the D3 branes.

\footnotetext{
${ }^{2}$ Given the limited available tools for dealing with sigma-models with RR backgrounds, the procedure outlined here seems at present the only precise method for extracting the cut-off dependence of the sigma-model expectation values. We should further mention that, in case the string theory under consideration is an orientifold compactification, one also needs to include world-sheets with an arbitrary number of cross-caps. These can be treated in a similar fashion as the open string holes and $\Phi$ operator insertions.
} 

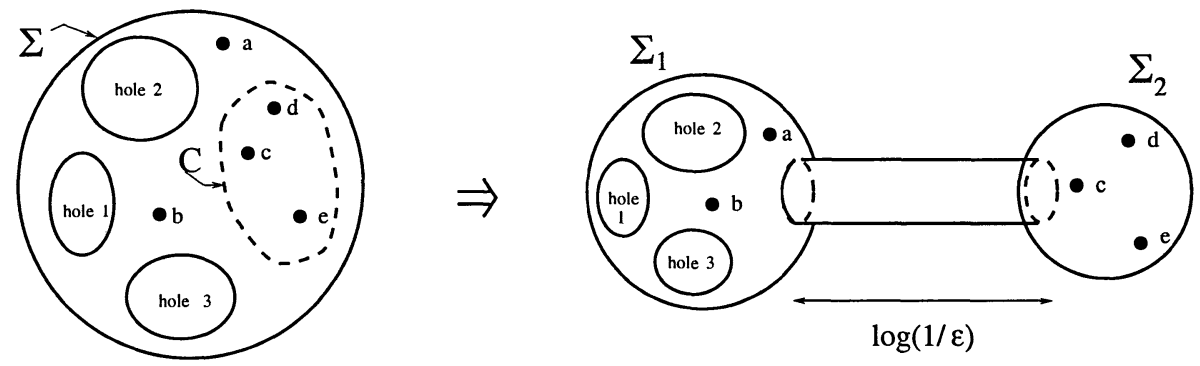

Fig. 2a and 2b. After expanding the sigma-model interaction term $\exp \Phi$, planar open string diagrams have in addition to holes also a number of insertions of the closed string operator $\Phi$ located at isolated punctures. The sigma model divergences correspond to the degeneration of non-contractible curves $C$ that separate off spheres with no holes but with a non-zero number of punctures.

It is now straightforward to determine the $\epsilon$ dependence of the total effective action. Each term $\left\langle(\Phi)^{k}\right\rangle_{\mathrm{n}}$ is given by an integral over the corresponding moduli space, whose only dependence on $\epsilon$ is via the restriction (11) on the geodesic lengths. Hence if we differentiate $\left\langle(\Phi)^{k}\right\rangle_{\mathrm{n}}$ with respect to $\epsilon$, the result is an integral over the boundary sub-space for which (at least) one of the contours $C$ has reached its minimal length $\ell(C)=\epsilon$. Now it is a well known fact that such a pinched surface is conformally equivalent to a surface for which the closed string propagator in the dual channel has acquired a large length proportional to $\log (1 / \epsilon)$. (See figs $1 \mathrm{~b}$ and $2 b$.) The partition function for this degenerate surface factorizes into a sum of products of two one-point functions defined on each half of the surface on each side of this long propagator. Concretely, writing the evolution operator along this long tube as

$$
\epsilon^{L_{0}+\bar{L}_{0}}=\frac{1}{2} \sum_{i}\left|O_{i}\right\rangle G^{i j}\left\langle O_{j}\right|,
$$

(here we assume that the set of states $\left|O_{i}\right\rangle$ forms a complete basis of closed string states) we can express the explicit $\epsilon$-dependence of the $\mathrm{n}$ loop partition function $\Gamma_{\mathrm{n}}$ as follows

$$
\epsilon \frac{\partial \Gamma_{\mathrm{n}}}{\partial \epsilon}=-\frac{1}{2} \sum_{0 \leq \mathrm{m} \leq \mathrm{n}} G^{i j} \frac{\partial \Gamma_{\mathrm{m}}}{\partial \phi^{i}} \frac{\partial \Gamma_{\mathrm{n}-\mathrm{m}}}{\partial \phi^{j}}
$$

The summation here runs over all possible ways of dividing the surface with $\mathrm{n}$ holes into two parts, as indicated in figs $1 \mathrm{~b}$ and $2 \mathrm{~b}$. 
The $\mathrm{m}=0$ and $\mathrm{m}=\mathrm{n}$ terms in eqn (16), the ones containing a factor $\partial \Gamma_{0} / \partial \phi^{i}$, describe the 'splitting off' of a sphere without holes as indicated fig $2 \mathrm{~b}$, and represent the scale dependence due to the sigma model divergences. ${ }^{3}$ Our method of renormalization of the sigma model is to absorb this particular type of divergence by means of the transition to renormalized couplings $\phi^{i}(\epsilon)$. In other words, the $\epsilon$ dependence of $\phi^{i}(\epsilon)$ should be such that the terms proportional to $\partial \Gamma_{0} / \partial \phi^{i}$ cancel in the total $\epsilon$-variation of $\Gamma_{n}$. From this we deduce that the renormalized couplings must satisfy (9) with

$$
\beta^{i}(\phi)=G^{i j} \frac{\partial \Gamma_{0}}{\partial \phi^{j}}
$$

Note that a relation of this form is indeed expected from the identification (14) of $\Gamma_{0}(\phi)$ with the classical closed string field theory action.

[ This renormalization procedure (17) is further motivated by the requirement that the eventual renormalized form of the RG-equation should be independent of the cut-off $\epsilon$. In particular the factorization metric $G^{i j}$ introduced in (15) should be cut-off independent. It seems reasonable to assume that the only divergences, that induce a renormalization of $G^{i j}$, are those of the sigma-model. In our regularization method, these arise from 'contact term' contributions produced when one or more of the $\Phi$-operator insertions get 'trapped' somewhere on the long tube in fig $2 \mathrm{~b}$. The effect of these terms is to replace the $L_{0^{-}}$ operators in (15) by those in the $\Phi$-background. The resulting condition of $\epsilon$-independence of $G^{i j}$ takes the form

$$
\epsilon \frac{\partial G^{i j}}{\partial \epsilon}+\beta^{k} \frac{\partial G^{i j}}{\partial \phi^{k}}-\Delta_{k}^{i} G^{k j}-\Delta_{k}^{j} G^{i k}=0 .
$$

with $\beta^{i}$ as in (17) and where $\Delta_{i}^{k}=\partial_{i} \beta^{k}$ denotes the matrix of conformal dimensions of the $O_{i}$ in the $\Phi$-background. ]

Via the definition of the total action $S$ as the sum of all $\Gamma_{n}$ 's, we can summarize the infinite set of relations (16) as a single non-linear

\footnotetext{
${ }^{3}$ Note that (via the presence of the kinetic term in (14)) $\partial \Gamma_{0} / \partial \phi^{i}$ starts out for small $\phi$ with a linear term proportional to $\Delta_{j}^{i} \phi^{j}$, with $\Delta_{j}^{i}$ the matrix of scaling dimensions of the $O_{i}$ (defined via $L_{0}\left|O_{i}\right\rangle=\Delta_{i}^{j}\left|O_{j}\right\rangle$ ). The corresponding explicit cut-off dependence of the correlators $\left\langle(\Phi)^{k}\right\rangle_{\mathrm{n}}$ arises from the local regularization of the individual $\Phi$ operator insertions.
} 
equation for $S$.

$$
\epsilon \frac{\partial S}{\partial \epsilon}=-\frac{1}{2} G^{i j} \frac{\partial S}{\partial \phi^{i}} \frac{\partial S}{\partial \phi^{j}},
$$

and we thus indeed obtain the announced form for the total FS scale invariance condition (10)

$$
\beta^{i}(\phi) \frac{\partial S}{\partial \phi^{i}}-\frac{1}{2} G^{i j} \frac{\partial S}{\partial \phi^{i}} \frac{\partial S}{\partial \phi^{j}}=0 .
$$

This equation, as well as our derivation, are strongly reminiscent of Polchinski's version of the Wilsonian renormalization group [11]. Indeed our UV-regulator (11) looks just like an IR cut-off on the (proper length of the) closed string propagator in the dual channel. It is not surprising therefore that the explicit $\epsilon$-dependence of $S$ looks just like (the classical limit of) the RG-equation derived in [12].

Finally, we remark that the total equation of motion for $S$ implies that on-shell

$$
G^{i j} \frac{\partial S}{\partial \phi^{j}}=\beta^{i}(\phi)+\sum_{\mathrm{n} \geq 1} \lambda^{\mathrm{n}} G^{i j} \frac{\partial \Gamma_{\mathrm{n}}}{\partial \phi^{j}}=0,
$$

which tells us that the deviation from scale-invariance coming from the sigma-model beta-functions cancels against that coming from the source terms due to the presence of the D3-branes, and/or from the open string loop contributions. ${ }^{4}$

\footnotetext{
${ }^{4}$ The term with $\mathrm{n}=1$ is a pure D3-brane source term; the other terms with $\mathrm{n}>1$ can either be viewed as higher order closed string source terms or as quantum corrections due to open string loops.
} 

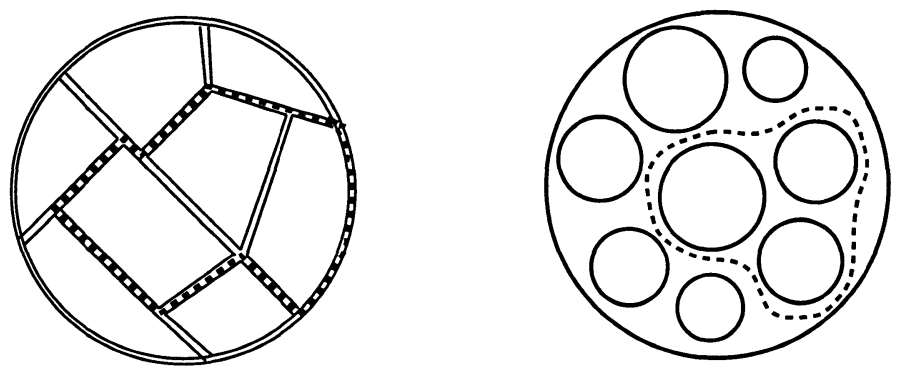

Fig. 3. In the zero slope limit $\alpha^{\prime} \rightarrow 0$, the open string loop diagram reduces to an ordinary planar Feynman graph of the low energy gauge theory. The restriction (11) on the minimal geodesic length of the noncontractible contour $C$ then translates into a lower bound on the sum of the Schwinger parameters $t_{i}$ of the propagators contained in $C$.

\section{Equation of Motion and Bogolyubov's Recursion Formula}

Although the above line of argument did not make any use of the AdS/CFT correspondence, it seems still quite essential that the planar diagrams were in fact string world sheets, so that one can easily visualize the transition from the open to the closed string channel. In principle, however, it should be straightforward (though presumably quite tedious) to explicitly take the $\alpha^{\prime} \rightarrow 0$ limit and translate all steps into purely field theoretic language. Here we limit ourselves to just a couple of remarks.

As indicated in fig 3, our regulatization method for the open string diagrams carries over quite directly to ordinary planar diagrams. Feynman diagrams of the low energy large $N$ gauge theory can also be written as integral over a "moduli space" of Schwinger parameters, parametrizing the proper length $t_{i}$ of the propagators. The UV regulator (11) thus amounts to the restriction that for any closed path $C$ on the graph (as in fig 3)

$$
\sum_{i \in C} t_{i}>\epsilon
$$

where the sum is over all propagators that make up the contour $C$. This restriction indeed renders the integral UV finite. 
We can now use a similar reasoning as above to try and extract the $\epsilon$ dependence, by explicitly differentiating the total integral over all Schwinger parameters with respect to the UV cut-off (22). The analog of the formula (15) should now be extracted from analyzing the UV limit of the one-loop gauge theory amplitude in a background large $N$ gauge-field $A$, with couplings $\phi^{i}$ turned on; equation (15) then corresponds to the fact that, to leading order in $1 / N$, this amplitude factorizes into a sum over gauge-invariant single-trace operators $O_{i}$.

Useful insight into how one should interpret the sigma model data contained in $\Phi$ is obtained by considering the equation of motion of the total effective action (16). It is possible to write it in the form of a recursion relation, by expanding the closed string background $\Phi$ in powers of the string coupling $\lambda$

$$
\Phi=\sum_{\mathrm{n} \geq 1} \lambda^{\mathrm{n}} \Phi_{\mathrm{n}}
$$

where $\Phi_{\mathrm{n}}$ is assumed to be independent of $\lambda$. The equation of motion of $\Phi_{\mathrm{n}}$

$$
\frac{\delta S}{\delta \Phi_{\mathrm{n}}}=0
$$

now takes the following form

$$
Q\left|\Phi_{\mathrm{n}}\right\rangle=[1]_{\mathrm{n}}+\sum_{1 \leq m \leq n} \sum_{\sum_{\mathrm{j}} \mathrm{j} k_{\mathrm{j}}=\mathrm{m}} \frac{1}{k_{1} ! \cdots k_{\mathrm{m}} !}\left[\left(\Phi_{1}\right)^{k_{1}} \ldots\left(\Phi_{\mathrm{m}}\right)^{k_{\mathrm{m}}}\right]_{\mathrm{n}-\mathrm{m}} .
$$

Here $[\ldots]_{\mathrm{n}}$ denotes the state associated to a surface as indicated in fig 4: the sphere with $n$ holes at the end of a tube with length $\log (1 / \epsilon)$, and with operator insertions specified by the (...). The above formula can be used to recursively construct $\Phi_{\mathrm{n}}$ from the previous $\Phi_{\mathrm{m}}$ 's with $\mathrm{m}<\mathrm{n}$. 


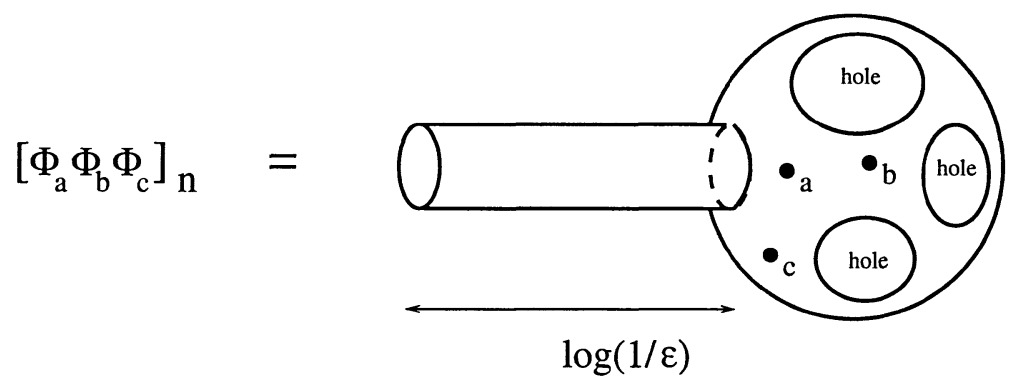

Fig. 4. This figure illustrates the notation used in (25): [ . . $]_{\mathrm{n}}$ denotes the state associated with the operators $(. .$.$) inserted on a sphere with$ $n$ holes at the end of a tube of length $\log (1 / \epsilon)$.

The field theoretic meaning of the above recursive form of the equation of motion is as follows: the term $\Phi_{\mathrm{n}}$ is associated with the nth order counter term designed to cancel the divergent contribution [ 1$]_{\mathrm{n}}$ of the $\mathrm{n}$-loop vacuum graph, that is left after subtracting all sub-divergences of lower order. There is indeed a strong resemblance between eqn (25) and the famous recursion relation due to Bogolyubov [13] for constructing the successive counterterms in the BPHZ renormalization method of QFT. We suspect that, by carefully taking the $\alpha^{\prime} \rightarrow 0$ limit of (25), this match can be made even more precise. ${ }^{5}$

\section{Conclusion: World-sheet vs Space-Time RG}

Our main result is equation (20) and the fact that it reproduces (at least in form and with the identification $\Gamma_{0}(\phi)=S_{E}(\phi)$ ) the flow equation (3) derived from classical 5-d supergravity. Both derivations have their limited range of validity: the AdS/CFT duality used in [6] is accurate only the regime of $\lambda \gg 1$, whereas the perturbative reasoning of this paper requires $\lambda \ll 1$. The correspondence between the two, however,

\footnotetext{
${ }^{5}$ It is also not entirely coincidental that the general solution to Bogolyubov's recursion relation, due to Zimmerman [14], is known as the forest formula. In the present context, a forest (a nested or disjoint set of sub-diagrams associated with nested or disjoint sub-divergences) in essence corresponds to a set of sub-trees of a closed string tree diagram.
} 
provides strong evidence that the same structure persists for all values of the 't Hooft coupling $\lambda$.

Equation (20) has an interpretation as both a world-sheet and spacetime $\mathrm{RG}$ relation. ${ }^{6}$ The first interpretation is manifest from our derivation, since the $\beta^{i}(\phi)$ represent the world-sheet scale dependence of the sigma-model couplings $\phi^{i}$. The second interpretation arises via the identification of space-time RG transformations with Weyl rescalings of the 4-d target-space metric $g^{\mu \nu}$. This motivates the following translation code between the world-sheet and space-time beta-functions (cf. [6])

$$
\begin{aligned}
& \beta_{\mathrm{wS}}^{i}=\gamma \beta_{\mathrm{ST}}^{i} \\
& \beta_{\mathrm{wS}}^{\mu \nu}=2 \gamma g^{\mu \nu}+\gamma \beta_{\mathrm{ST}}^{\mu \nu}
\end{aligned}
$$

where $\beta_{W S}^{\mu \nu}$ denotes the world-sheet beta-function of the target-space metric $g^{\mu \nu}$. Via the above translation, the invariance condition (20) takes the form of a quite conventional RG-flow equation in space-time.

Note that in this correspondence the world-sheet and space-time scales are proportional to each other, so UV and UV are mapped to each other as well as IR to IR. We further see from (27) that the prefactor $\gamma$, which determines the relative normalization of the world-sheet and space-time beta-functions, is (via eqn (17)) proportional to the cosmological term in $\Gamma_{0}(\phi)$. This term, generated by the RR background as well as by other expectation values, is typically of order the fundamental string scale and can be even larger. Although, as seen from [6], the interpretation of the 4-d scale as a holographic extra dimension is expected to break down in this regime, we have shown here that the RG-invariance (20) of $S$ is nonetheless preserved.

\section{Acknowledgements}

This work is supported by NSF-grant 98-02484. We would like to thank M. Berkooz, R. Dijkgraaf, G. Lifschytz, V. Periwal, A. Polyakov and

\footnotetext{
${ }^{6}$ Suggestions as well as concrete proposals towards a correspondence between world-sheet and space-time RG were made by many authors, including in [2], [9], $[12],[15]$ and $[16]$.
} 
E. Verlinde for useful discussions. J.K. is supported by the Natural Sciences and Engineering Research Council of Canada.

\section{References}

[1] J. Maldacena, The Large $N$ Limit of Superconformal Field Theories and Supergravity, Adv. Theor. Math. Phys., 2 (1998), 231, hep-th/9711200.

[2] S. Gubser, I. Klebanov, and A. Polyakov, Gauge Theory Correlators from Non-Critical String Theory, Phys. Lett., B428 (1998), 105, hep-th/9802109.

[3] E. Witten, Anti-de Sitter Space and Holography, Adv. Theor. Math. Phys., 2 (1998), 253, hep-th/9802150.

[4] H. Verlinde, Holography and Compactification, hep-th/9906182.

[5] L. Randall and R. Sundrum, A Large Mass Hierarchy from a Small Extra Dimension, Phys. Rev. Lett., 83 (1999), 3370, hep-ph/9905221; An Alternative to Compactification, Phys. Rev. Lett., 83 (1999), 4690, hep-th/9906064.

[6] E. Verlinde, and H. Verlinde, RG flow, Gravity and the Cosmological Constant, hep-th/9912018;

see also J. de Boer, E. Verlinde, and H. Verlinde, On the Holographic Renormalization Group, hep-th/9912012.

[7] W. Fischler and L. Susskind, Dilaton Tadpoles, String Condensates and Scale Invariance, Phys. Lett., B173 (1986), 262;

C.G. Callan, C. Lovelace, C.R. Nappi, and S.A. Yost, String Loop Corrections to Beta Functions, Nucl. Phys., B288 (1987), 525; Adding Holes and Crosscaps to the Superstring, Nucl. Phys., B293 (1987), 83; Loop Corrections to Superstring Equations of Motion, Nucl. Phys., B308 (1988), 221;

J. Polchinski and Y. Cai, Consistency of Open Superstring Theories, Nucl. Phys., B296 (1988), 91.

[8] D. Berenstein and R.G. Leigh, Superstring Pertrubation Theory and Ramond-Ramond Backgrounds, Phys. Rev., D60 (1999), 106002, hep-th/9904104. 
[9] R. Brustein and S.P. De Alwis, Renormalization Group Equation and Nonperturbative Effects in String Field Theory, Nucl. Phys., B352 (1991), 451;

R. Brustein and K. Roland, Space-Time Versus World Sheet Renormalization Group Equation in String Theory, Nucl. Phys., B372 (1992), 201.

[10] B. Zwiebach, Closed String Field Theory: Quantum Action and the BV Master Equation, Nucl. Phys., B390 (1993), 33, hep-th/9206084.

[11] J. Polchinski, Renormalization and Effective Lagrangians, Nucl. Phys., B231 (1984), 269.

[12] A. Polyakov, last of A Few Projects in String Theory, hep-th/9304146.

[13] N. Bogolyubov and D. Shirkov, Introduction to the Theory of Quantized Fields, Interscience, New York, 1959.

[14] W. Zimmermann, Ann. of Phys., 77, (1973), 536.

[15] E. Alvarez and C. Gomez, Non-Critical Confining Strings and the Renormalization Group, hep-th/9902012.

[16] C. Schmidhuber, AdS-Flows and Weyl Gravity, hep-th/9912155. 